\title{
Modeling and Simulation of Predictive Maintenance Scheme for High Speed Railway Vehicles
}

\author{
Khurram Shaikh*, Imtiaz Hussain Kalwar, Bhawani Shankar Chowdhry, Kamran Kazi and Burhan \\ Aslam Arain \\ Department of Electronic Engineering, MUET, Jamshoro; khuram.shaikh@admin.muet.edu.pk
}

\begin{abstract}
This paper presents a signal based predictive maintenance strategy for high speed railway vehicle having solid axle wheelsets. Several railway operators still rely on manual inspection of rolling stock, which is costly and inaccurate. In this research work an accurate and cost effective method is proposed to monitor the condition of the wheelset of in service vehicles. In proposed method inertial sensors are used to measure the lateral and yaw dynamics of the wheelset. The condition of the wheelset is then predicted indirectly by exploiting the measured dynamic response. To show the effectiveness of the proposed method a simulation model of the proposed scheme is developed in Matlab/Simulink. The simulation results affirm the proposed idea.
\end{abstract}

Keywords: Predictive Maintenance, Railway Vehicle, Wheelset

\section{Introduction}

In railway transport nowadays wheelset maintenance is usually scheduled based. However, the wheel tread does not wear down consistently over the same period of time. The wearing of wheel tread depends upon several factors such as environmental conditions (friction level on tracks), track condition even on the number of passengers travelling on the train. The involvement of the uncertain parameters such as weather and environmental conditions makes it very difficult to perform the maintenance on a fix schedule. Therefore, in this research work a novel technique is proposed that can continuously monitor the condition of the railway wheelset of an in-service vehicle and will notify if maintenance is required.

The railway wheelset running on tracks is a complicated mechanical system because its dynamics are governed by several factors such as nonlinear creep forces generated at wheel-rail contact patch and unpredictable fluctuations in the contact conditions. Beside these reasons, vehicle speed, suspension and flange stiffness also affects the dynamics of the railway wheelset ${ }^{1}$. Among several other factors, wheelset conicity plays a vital role in the wheelset dynamics. Even a small variation in conicity level can result in serious damage to the wheel tread and track. Several vehicle derailments have taken place due to wheel conicity, one of them occurred in March 2006, when two wheelsets of tram of the Manchester Metrolink system as it was approaching the street running section of the network at Long Millgate, near Victoria Station. There were no casualties and the railcar was not impaired ${ }^{2}$.

Several techniques have been proposed to address this issue. $\operatorname{In}^{\frac{3-6}{6}}$ model based techniques are proposed to monitor the creep forces and adhesion level at the wheelrail interface. However, the fundamental requirement of a model based techniques is an accurate mathematical model. The dynamics of the wheelset are highly nonlinear and their dependency on uncertain parameters such as weather and environmental condition makes it almost impossible to derive an accurate mathematical model. Signal based methods are comparatively popular in railway condition monitoring.

Several signal based methods are also proposed to monitor the condition of railway rolling stock. In signal based method sensors are employed in different parts of the vehicle such as axle and bogies frame. The captured

*Author for correspondence 
signals are further analyzed using different signal processing techniques to detect any abnormality in the operation. A vibration based condition monitoring method is proposed in $\mathrm{n}^{7}$ to evaluate of the bogie performance of freight train. Similar kinds of methods are also proposed in $\frac{8.9}{}$ to monitor the condition of railway suspension system. A laser based direct conicity measurement method is presented $\mathrm{in}^{\underline{10}}$. The problem with this method is that an accurate measurement requires precise alignment of laser which in the presence of vibrations is not possible.

Many researchers around the world have worked on this issue using and have proposed several different techniques. None of these techniques has been able to tackle this problem completely because railway dynamics are extremely complicated. It is hard to predict a cause of failure in presence of non-linearities and uncertainties such as non-linear wheel-rail contact mechanics and uncertain behavior of adhesion coefficient. In this research work a new method is proposed to solve this issue. The proposed technique makes use of inertial sensors mounted on axle to capture the kinematic oscillations. Further analysis is carried out to determine the conicity level from the spectral properties of the captured signal.

\section{Modeling of Wheelset Dynamics}

In this research work a single unconstrained solid axle wheelset is considered to demonstrate the potential of the proposed idea. Figure 1 shows the basic configuration of a bogie of modern passenger carrying railway vehicle. One railway car consists of two bogies and in each bogie there are two wheelsets ${ }^{11}$. The wheelsets are connected via suspension elements with the bogie frame.

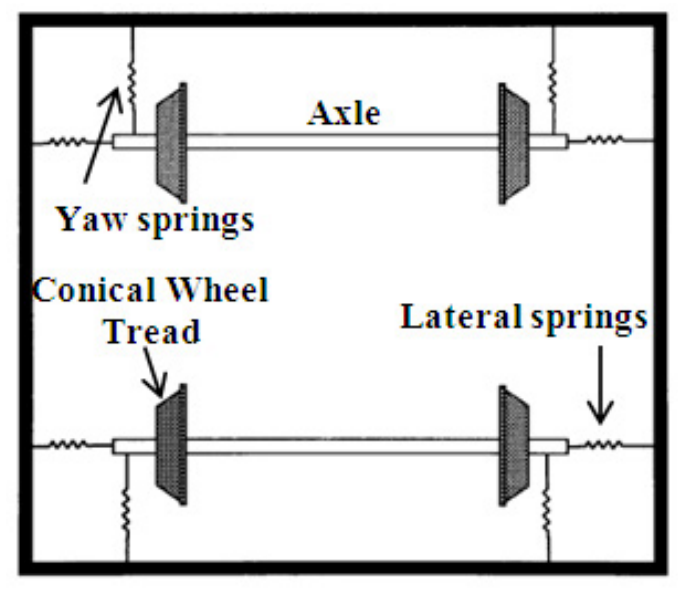

Figure 1. Railway bogie.
The proposed idea is based on the fact that an unconstrained wheelset exhibits kinematic oscillations at non-zero speeds. The frequency of oscillation is given by Klingel's formula ${ }^{12}$.

$$
f=\frac{v_{s}}{2 \pi} \sqrt{\frac{\gamma_{W}}{L_{g} r_{o}}}
$$

A simulation model is developed using a simplified mathematical model (eq-2) presented in [4] to simulate the behavior of a Railway vehicle. In mathematical model only lateral and yaw dynamics are considered for preliminary study which can be easily extended to consider other relevant dynamics.

$$
\left[\begin{array}{c}
\dot{y}_{w} \\
\dot{\emptyset}_{w} \\
\ddot{y}_{w} \\
\ddot{\emptyset}_{w}
\end{array}\right]=\left[\begin{array}{cccc}
0 & 0 & 1 & 0 \\
0 & 0 & 0 & 1 \\
0 & \frac{2 f_{22}}{m_{w}} & -\frac{2 f_{22}}{m_{w}} & 0 \\
-\frac{2 L_{g} \gamma_{w} f_{11}}{r_{o} I_{w}} & -\frac{k_{w}}{I_{w}} & 0 & -\frac{2 L_{g}^{2} f_{11}}{v_{v} I_{w}}
\end{array}\right]\left[\begin{array}{c} 
\\
y_{w} \\
\Psi_{w} \\
\dot{y}_{w} \\
\ddot{\emptyset}_{w}
\end{array}\right]+\left[\begin{array}{c} 
\\
0 \\
0 \\
0 \\
\frac{2 L_{g} \gamma_{w} f_{11}}{r_{o} I_{w}}
\end{array}\right] y_{t}
$$

In equation-2 $\gamma_{W}$ represents conicity of wheelset. If other parameters are kept constant during the simulation than it isclear from the equation- 2 that the dynamic behavior is only changed when the conicity of the wheelset is changed. Simulation model shown in following figure is developed using equation-2.

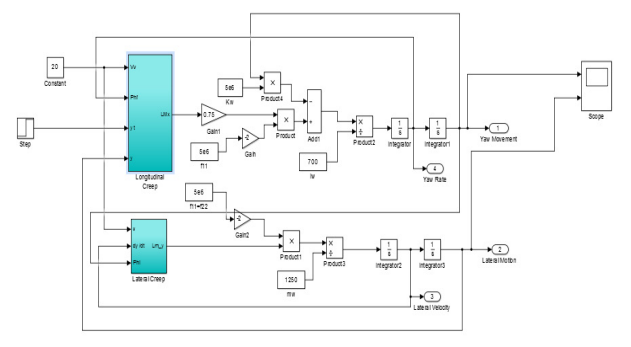

Figure 2. Simulation model of railway wheelset.

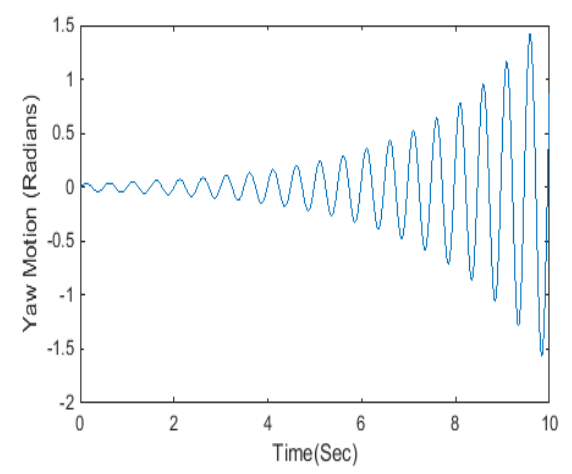

Figure 3. Step response of constrained wheelset (Yaw Motion). 


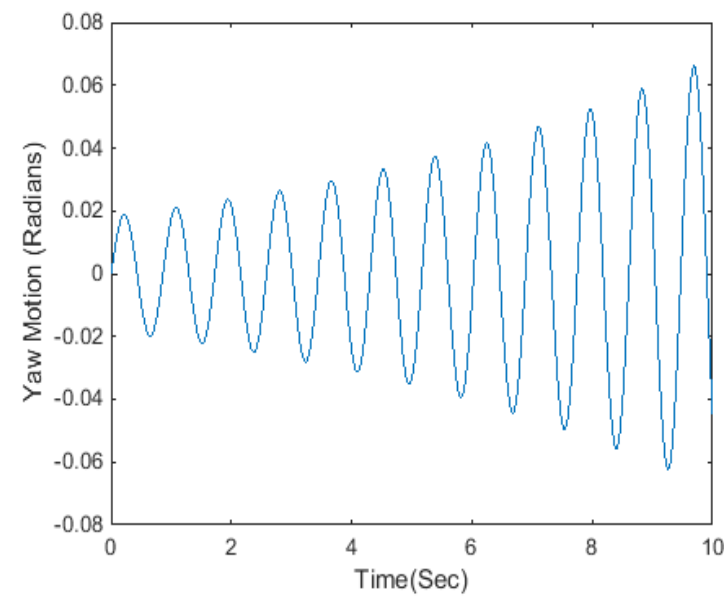

Figure 4. Step response of unconstrained wheelset (Yaw Motion).

When the wheelset is constrained (i.e.) the wheelset stabilizes itself after an accidental lateral force is induced from the track. This phenomenon is shown in Figure 3.

Whereas, when the wheelset is unconstrained (i.e. $k_{w}=\mathbf{0}$ )the wheelset exhibits kinematic oscillations as shown in Figure 4. The frequency of oscillations can be calculated by using Klingel's formula given in equation 1 .

If all other parameters in equation-1 are kept constant, then the frequency of oscillation will only depend upon conicity of the wheelset. Therefore, the simulations are carried out at different conicity levels and the results of simulation are given.

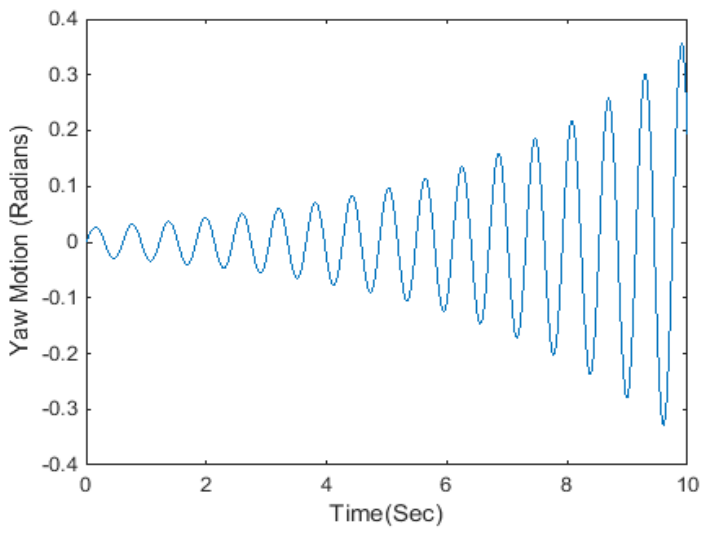

Figure 5. Step response of unconstrained wheelset ( $\gamma_{w}=0.05$ ).

If all other parameters in equation-1 are kept constant, then the frequency of oscillation will only depend upon conicity of the wheelset. Therefore, the simulations are carried out at different conicity levels and the results of simulation are given.

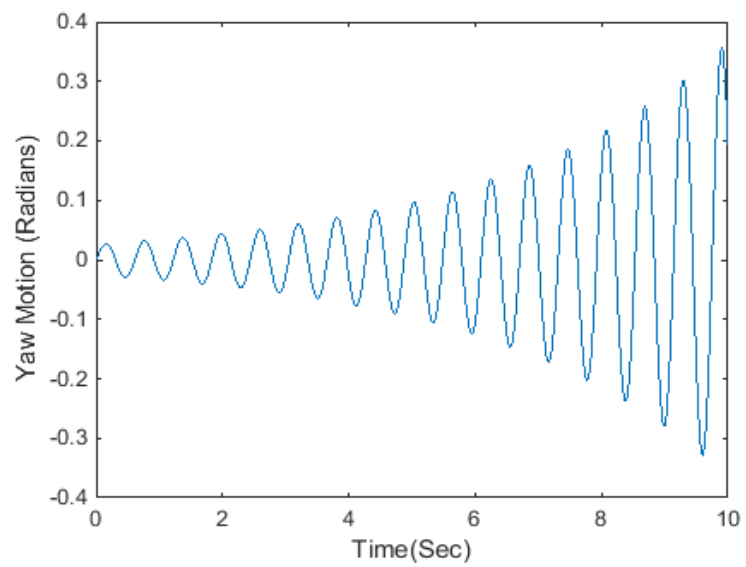

Figure 6. Step response of unconstrained wheelset ( $\left.\gamma_{w}=0.1\right)$.

Figure 5 shows the yaw motion of unconstrained wheelset when conicity level is very low. If the conicity is further decreased frequency of oscillation is also decreased. Decreasing conicity below 0.05 means the wheel tread is worn out and has almost become cylindrical in shape. Cylindrical wheel tread does not have the tendency to align itself back at the center position. Therefore, it is necessary in proper operation of railway that the certain conicity level is maintained to ensure safe and comfortable operation of railway transport.

In Figure 6 conicity is increased to 0.1 which result in the increase of frequency of oscillation.

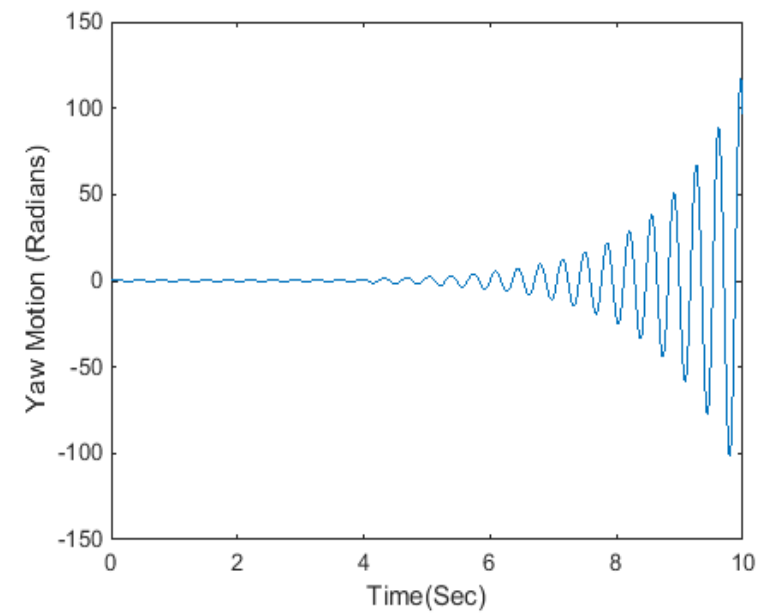

Figure 7. Step response of unconstrained wheelset ( $\gamma_{w}=0.2$ ). 


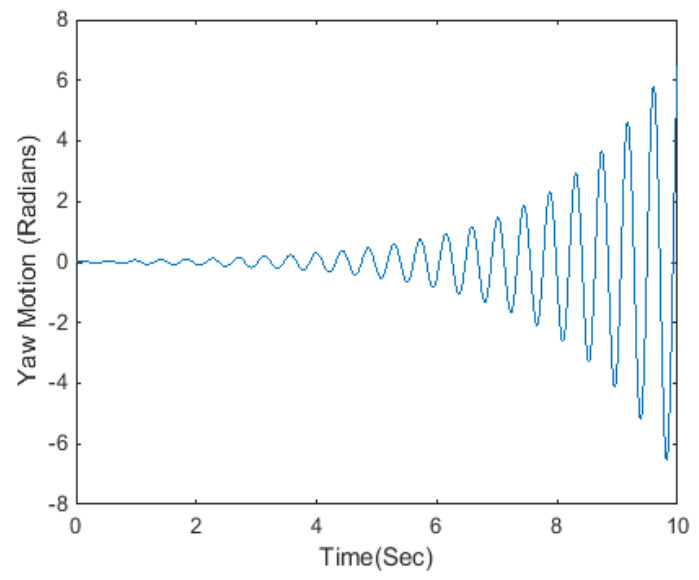

Figure 8. Step response of unconstrained wheelset ( $\left.\gamma_{w}=0.3\right)$.

In Figure 7, conicity is further increased to 0.2 which is reflected in the frequency of oscillation.

As the conicity level is increased beyond 0.2 the high frequency vibrations are produced which may cause discomfort to passengers, permanent mechanical component failure and huge noise as the train passes along the track.

\section{Discussions}

From the above results it is evident that certain range of conicity level is suitable for noise free, vibration free and comfortable railway operation. If the conicity level of in-service vehicle is deviated from the allowable range, then the consequences can be severe. Therefore, a new method is proposed here which can indirectly identify the changes in conicity level by analyzing the changes in spectral properties of inertial signals. The block diagram of the proposed scheme is shown in Figure 9.

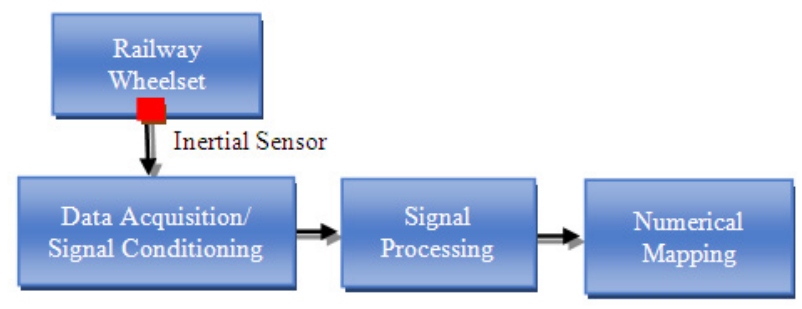

Figure 9. Block diagram of proposed idea.

The signal captured by inertial sensors is first preprocessed from signal conditioning block. This block removes noise content present in the signal and processes the data further to signal processing block. Signal processing block then computes the fast Fourier Transform of the inertial signal. The simulation results are obtained at different conicity levels and are presented.

Figure 10 shows the FFT of kinematic oscillations when conicity level is low. The frequency of oscillation is nearly $0.5 \mathrm{~Hz}$. In Figure 11 when conicity is doubled the frequency of oscillation is increased $(\mathrm{f}=0.8 \mathrm{~Hz})$.

If the conicity level is further increased the frequency of oscillation is also increased as shown in Figures 12 and 13. In Figure 12 frequency of oscillation is $1.16 \mathrm{~Hz}$. When the conicity level is 0.3 the frequency of oscillation is also increased to $1.4 \mathrm{~Hz}$.

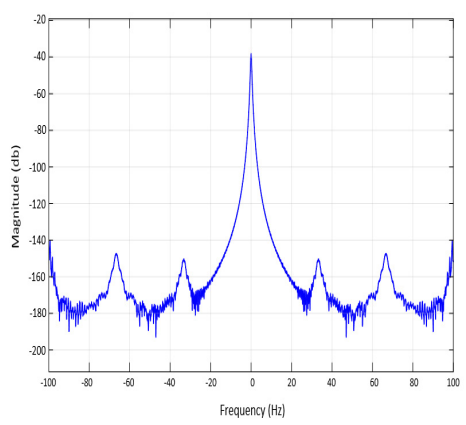

Figure 10. Kinematic oscillations of wheelset with $\gamma=0.05$ and velocity $=10$.

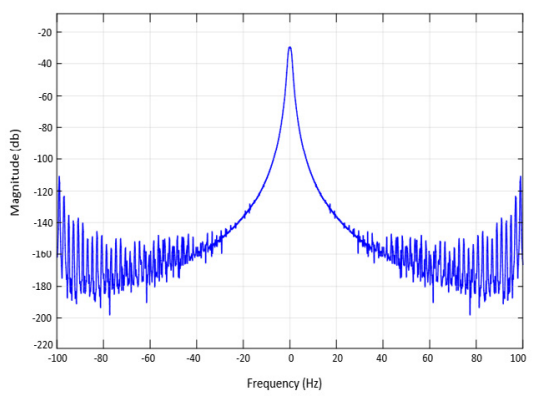

Figure 11. Kinematic oscillations at conicity $(\gamma)=0.1$ and velocity $=10$.

The simulation results obtained above are summarized in Table 1. In order to derive a numerical relationship between frequency of oscillation and conicity level, the obtained results are plotted with frequency of oscillation on $\mathrm{x}$-axis and corresponding conicity level on $\mathrm{y}$-axis as shown in Figure 14. 


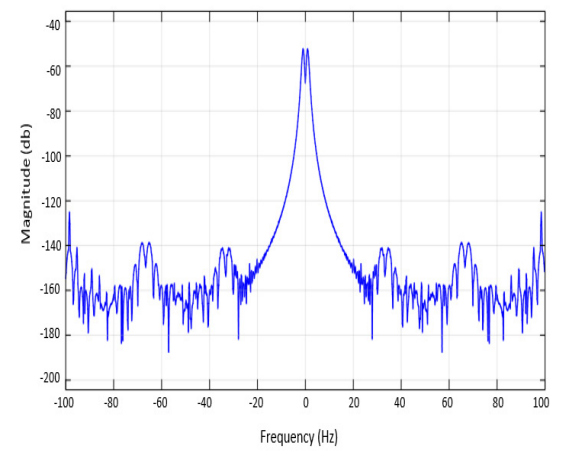

Figure 12. Kinematic oscillations at conicity $(\gamma)=0.3$ and velocity $=10$.

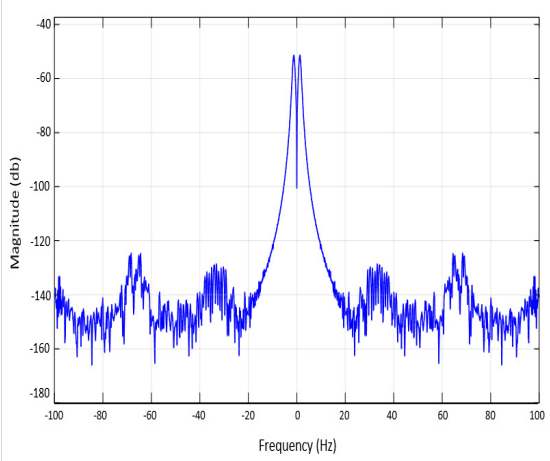

Figure 13. Kinematic oscillations at conicity $(\gamma)=0.2$ and velocity $=10$.

Table 1. Frequency vs conicity level

\begin{tabular}{|l|l|l|}
\hline S. No & Conicity & Frequency $\mathbf{( H z})$ \\
\hline 1 & 0.05 & 0.5 \\
\hline 2 & 0.1 & 0.8 \\
\hline 3 & 0.2 & 1.1 \\
\hline 4 & 0.3 & 1.4 \\
\hline 5 & 0.5 & 1.8 \\
\hline 6 & 0.6 & 2.0 \\
\hline
\end{tabular}

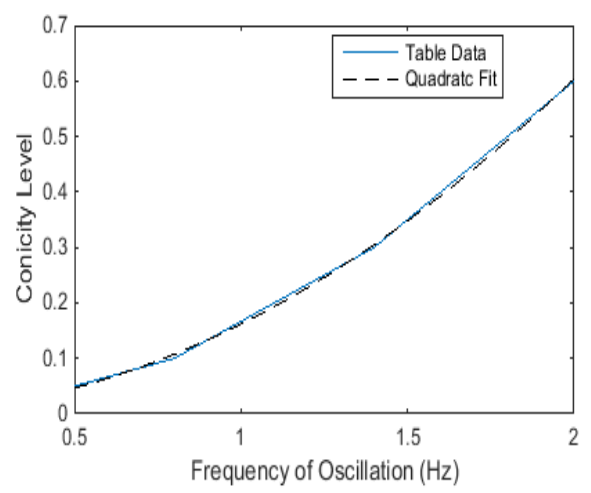

Figure 14. Frequency vs conicity level.
The simulation results obtained above are summarized in table-1. In order to derive a numerical relationship between frequency of oscillation and conicity level, the obtained results are plotted with frequency of oscillation on $\mathrm{x}$-axis and corresponding conicity level on $\mathrm{y}$-axis as shown in Figure 14.

From the graph a quadratic relationship is obtained between frequency of oscillation and conicity level.

$$
\gamma_{w}=0.14 f^{2}+0.018 f+0.0026
$$

Using equation- 3 the conicity level can be easily identified from the spectral properties of the inertial signal.

\section{Conclusion and Future Work}

The technological advancement has put immense pressure on modern railway transport to increase the safety and reliability of the transport. Plenty of research work is being carried out to resolve the issues related to maintenance of railway running gear. In this paper a new method is proposed to eliminate the possibility of under or over maintenance of mechanical parts. The presented results show great agreement with the proposed idea.

However, the work presented here is a preliminary research work. Further modifications are possible to improve the simulation results by considering the complete bogie model. Also the signal processing technique used here is very basic and not suitable for real time signals. Another advanced signal processing method such as wavelet transform or Hilbert Transform can be used as replacement of FFT.

\section{Acknowledgement}

This project is funded by Higher Education Commission (HEC) of Pakistan and the work is carried out at Top Quality Centralized Instrumentation Center (TQCIC), at Mehran University of Engineering and Technology, Jamshoro.

\section{References}

1. Knudsen C, Slivsgaard E, Rose M, True H, Feldberg R. Dynamics of a model of a railway wheelset. Nonlinear Dynamics. 1994 Sep; 6(2):215-36.

2. Derailment at Long Millgate, Manchester. Rail Accident Investigation Branch, Derby, United Kingdom, Rep. 08/2007; 2007 Apr. 
3. Hazlina S, Rubiyah Y. Wheel-rail contact parameters estimation for a conical solid-axle railway wheelset using least-absolute error with variable forgetting factor method. ICIT 2009.IEEE International Conference on Industrial Technology (2009), Gippsland, VIC; 2009. p. 1-6. DOI: 10.1109/ICIT.2009.4939485.

4. Imtiaz H, Mei TX, Mohammad M. Real time estimation of the wheel-rail contact conditions using multi-Kalman filtering and fuzzy logic. 2012 UKACC International Conference on Control (CONTROL), Cardiff, United Kingdom; 2012. p. 691-6. DOI: 10.1109/CONTROL.2012.6334713.

5. Christopher PW, Roger MG, Roger D. Wheel-rail profile condition monitoring. UKACC International Conference on Control, Conventry, United Kingdom; 2010. p. 1-6. DOI: 10.1049/ic.2010.0448.

6. Arthur A, Crinela P, Andrew B. A new method for modelling and simulation of the dynamic behaviour of the wheel-rail contact. International Journal of Automation and Computing. 2012 Jun; 9(3):237-47.

7. Parham S, Dan M, Brad H, Andrew S. Estimation of bogie performance criteria through on-board condition monitoring. Annual Conference of the Prognostics and Health Management Society, Fort Worth Texas, United States of America; 2014.
8. Weston PF, Roberts C, Goodman CJ, Ling CS. Condition monitoring of railway track using in-service trains. The Institution of Engineering and Technology International Conference on Railway Condition Monitoring, Birmingham, United Kingdom; 2006. p. 26-31.

9. Hitoshi T, andHirotaka M. Condition monitoring of railway vehicle suspension using adaptive multiple model approach. Proceedings of Control Automation and Systems (ICCAS), 2010 International Conference, Gyeonggi-do, South Korea; 2010. p. 584-9.

10. He P, You Z, Yang X. Research on application of synchronous sampling in high precision laser. Proceedings of Instrumentation and Measurement Technology, IMTC 04. Como, Italy. 2004; 3:2353-6. DOI: 10.1109/ IMTC.2004.1351565.

11. Hussain I, Halepoto IA, Kumar W, Kazi K. Anti-slip traction control of railway vehicle based on estimated wheel-rail contact condition. Sindh University Research Journal (Science Series). 2013; 45(2):373-8.

12. Wickens AH. Fundamentals of rail vehicle dynamics: guidance and stability. Taylor \& Francis; 2003. 